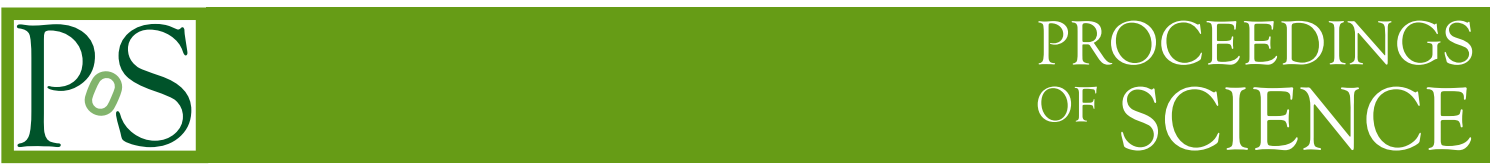

\title{
Large-scale simulations with chiral symmetry
}

\author{
JLQCD Collaboration: T. Kaneko ${ }^{* a, b \dagger}$, S. Aoki ${ }^{c}$, G. Cossu $^{a}$, H. Fukaya ${ }^{d}$, \\ S. Hashimoto ${ }^{a, b}$, J. Noaki ${ }^{a}$
}

${ }^{a}$ High Energy Accelerator Research Organization (KEK), Ibaraki 305-0801, Japan

${ }^{b}$ School of High Energy Accelerator Science, The Graduate University for Advanced Studies

(Sokendai), Ibaraki 305-0801, Japan

c Yukawa Institute for Theoretical Physics, Kyoto University, Kyoto 606-8502, Japan

${ }^{d}$ Department of Physics, Osaka University, Osaka 560-0043, Japan

We carry out a comparative study among five-dimensional formulations of chirally symmetric fermions about the algorithmic performance, chiral symmetry violation and topological tunneling to find a computationally inexpensive formulation with good chiral symmetry. With our choice of the lattice action, we have launched large-scale simulations on fine lattices aiming at a precision study of light and heavy quark physics. We report on the comparative study, current status of the large-scale simulations, and preliminary results on the residual quark mass and auto-correltion.

31st International Symposium on Lattice Field Theory - LATTICE 2013

July 29 - August 3, 2013

Mainz, Germany

\footnotetext{
*Speaker.

†E-mail: takashi.kaneko@kek.jp
} 


\section{Introduction}

In the last several years, we performed an extensive study of QCD vacuum and light hadron physics by using the overlap action which exactly preserves chiral symmetry [1]. Our next target is a precision study of heavy flavor physics in collaboration with flavor factory experiments, such as the SuperKEKB / Belle II experiment, for a stringent test of the Standard Model.

Since the overlap action is computationally too expensive to simulate small lattice spacings $a \ll m_{c}^{-1}$ on reasonably large lattices, we carried out a systematic comparative study of a class of five-dimensional formulations that approximately satisfy the Ginsparg-Wilson relation to construct a computationally cheap formulation with good chiral symmetry. In this article, we report on the comparative study and the status of the on-going large-scale simulations with our choice of the lattice action.

\section{Comparative study}

We test five-dimensional fermion formulations [2] in this comparative study. The four-dimensional effective Dirac operator is given by

$$
\frac{1+m_{q}}{2}+\frac{1-m_{q}}{2} \gamma_{5} \varepsilon_{M}\left(H_{M}\right),
$$

where the Hermitian kernel operator $H_{M}$ and the approximation of its sign function $\varepsilon_{M}$ can be chosen by tuning parameters appearing in the five-dimensional Dirac operator. Popular choices of $H_{M}$ are the Wilson kernel $H_{W}=\gamma_{5} D_{W}$, where $D_{W}$ is the Wilson-Dirac operator, for the overlap fermions, and the Shamir kernel $H_{T}=\gamma_{5} D_{W} /\left(2+D_{W}\right)$ for the standard domain-wall fermions. We also test a scaled Shamir kernel $2 H_{T}$ [2]. While $2 H_{T}$ has the same condition number as $H_{T}$, its lowlying eigenvalues are scaled up by a factor of 2 . These kernels are combined with the Zolotarev $\left(\varepsilon_{Z}\right)$ and polar decomposition $\left(\varepsilon_{p}\right)$ approximations. By applying up to 6 level stout smearing [3] $\left(N_{\mathrm{smr}}=0,3,6\right)$, we test 8 different formulations listed in Table 1 .

Table 1: Simulation setup in our comparative study. The first three columns show our choices of the fivedimensional formulation: the number of smearing $N_{\mathrm{smr}}$, kernel operator $H_{M}$ and sign function approximation $\varepsilon_{M}$. We also list simulation parameters, namely $\beta$ and the bare quark mass in lattice units $a m_{u d}$, as well as results for $a^{-1}$ and $M_{\pi}$.

\begin{tabular}{lll|ll|ll}
\hline$N_{\mathrm{smr}}$ & $H_{M}$ & $\varepsilon_{M}$ & $\beta$ & $a^{-1}[\mathrm{GeV}]$ & $a m_{u d}$ & $M_{\pi}[\mathrm{MeV}]$ \\
\hline \hline 0 & $H_{W}$ & $\varepsilon_{Z}$ & 4.27 & $1.98(6)$ & $0.0095,0.0060,0.0035$ & $463(17), 375(17), 346(25)$ \\
\hline 0 & $H_{T}$ & $\varepsilon_{Z}$ & 4.11 & $1.92(6)$ & $0.0200,0.0120,0.0065$ & $543(18), 419(15), 318(15)$ \\
\hline 0 & $H_{T}$ & $\varepsilon_{p}$ & 4.11 & $1.97(5)$ & $0.0200,0.0090,0.0040$ & $623(19), 483(16), 400(15)$ \\
\hline 0 & $2 H_{T}$ & $\varepsilon_{p}$ & 4.11 & $1.94(6)$ & $0.0200,0.0120,0.0065$ & $554(18), 434(17), 356(16)$ \\
\hline \hline 3 & $H_{W}$ & $\varepsilon_{Z}$ & 4.29 & $1.94(6)$ & $0.0145,0.0090,0.0050$ & $472(18), 401(17), 330(17)$ \\
\hline 3 & $H_{T}$ & $\varepsilon_{p}$ & 4.18 & $2.00(8)$ & $0.0250,0.0170,0.0090$ & $534(23), 423(20), 374(23)$ \\
\hline 3 & $2 H_{T}$ & $\varepsilon_{p}$ & 4.18 & $2.06(9)$ & $0.0250,0.0170,0.0090$ & $524(24), 469(24), 364(25)$ \\
\hline \hline 6 & $2 H_{T}$ & $\varepsilon_{p}$ & 4.18 & $2.11(6)$ & $0.0250,0.0170,0.0090$ & $511(17), 430(16), 337(20)$ \\
\hline
\end{tabular}



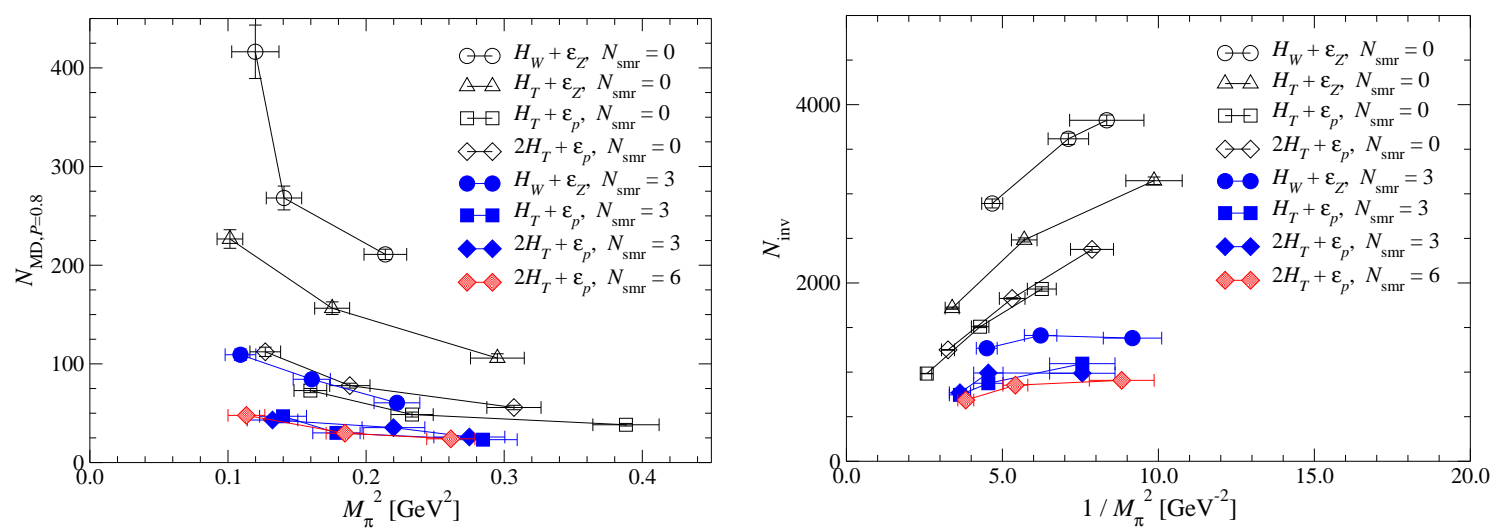

Figure 1: Left panel: number of MD steps $N_{\mathrm{MD}, P=0.80}$ to attain $80 \%$ acceptance rate. Data for different formulations are plotted in different symbols as a function of $M_{\pi}^{2}$. Right panel: CG iteration count $N_{\text {inv }}$ as a function of $M_{\pi}^{-2}$.
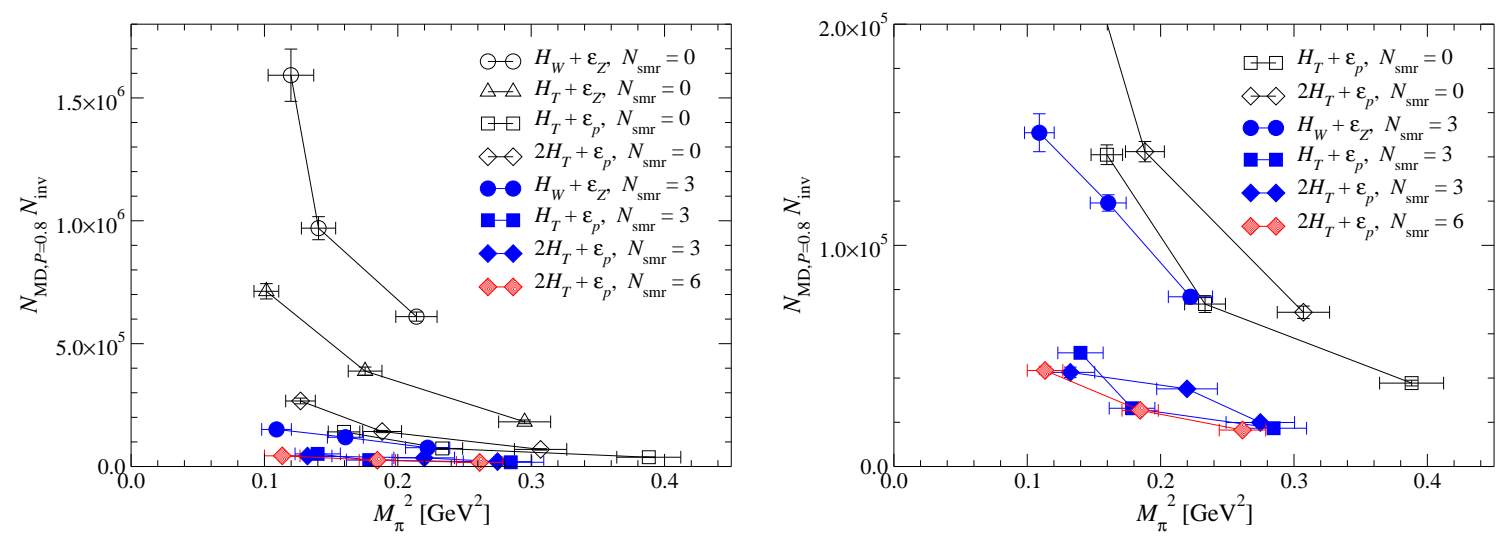

Figure 2: A measure of CPU cost per HMC trajectory $N_{\mathrm{MD}, P=0.80} N_{\mathrm{inv}}$. The left panel shows all data, whereas the right panel is an enlargement of a region of small $N_{\mathrm{MD}, P=0.80} N_{\mathrm{inv}}$ to focus on computationally cheaper formulations.

We carry out numerical simulations of two-flavor QCD by using these formulations and the tree-level Symanzik gauge action to study the performance of the Hybrid Monte Carlo (HMC) algorithm, chiral symmetry violation and topological tunneling. On a $16^{3} \times 32$ lattice, we simulate three pion masses in the range of $300 \lesssim M_{\pi}[\mathrm{MeV}] \lesssim 600$ at a single lattice cut-off around $a^{-1} \simeq$ $2 \mathrm{GeV}$. The fifth dimensional size is set to $N_{5}=12$. We set the range of the Zolotarev approximation $\varepsilon_{Z}(x)$ to $x \in[0.2,7.0]([0.4,7.0])$ for $H_{W}$ without (with) smearing, and $[0.1,1.5]$ for $H_{T}$. Our statistics are 1,000 trajectories in each simulation. Parameters and results for $a^{-1}$ and $M_{\pi}$ are summarized in Table 1, where $r_{0}=0.462(11)(4) \mathrm{fm}$ [4] is used as input to fix $a$.

In each simulation, we keep the acceptance rate of $P \simeq 0.7-0.9$ using a moderately small step size $\Delta \tau$ for the Molecular Dynamics (MD) integration. The number of the MD steps to attain a reference value $P=0.8$, which is denoted by $N_{\mathrm{MD}, P=0.80}$ in the following, is estimated from the relations holding at small $\Delta \tau$

$$
P=\operatorname{erfc}\left(\frac{1}{2} \sqrt{\langle\Delta H\rangle}\right), \quad\langle\Delta H\rangle \propto \Delta \tau^{4}
$$



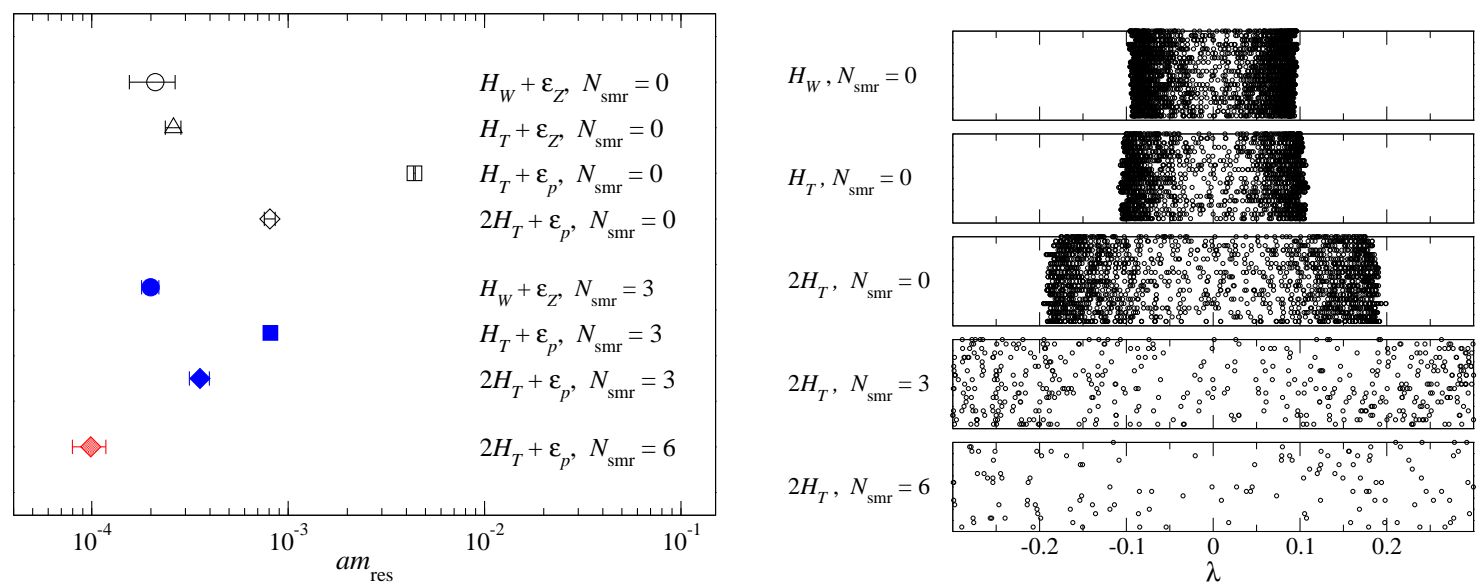

Figure 3: Left panel: a comparison of bare residual quark mass in lattice units, $a m_{\mathrm{res}}$. Right panels: distribution of eigenvalue $\lambda$ for several choices of the kernel operator. Note that we only plot the lowest $100-150$ eigenvalues for thin-link kernels $\left(N_{\mathrm{smr}}=0\right)$.
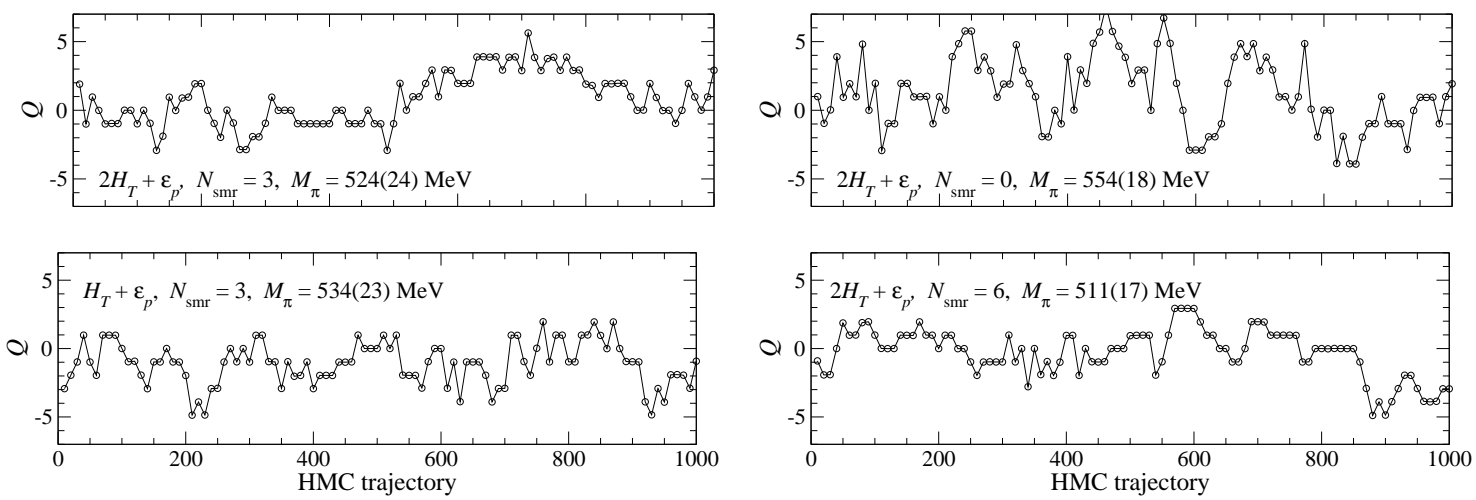

Figure 4: Monte Carlo history of topological charge. Left panels compare data for the Shamir-type kernels $\left(2 H_{T}\right.$ and $\left.H_{T}\right)$ with $\varepsilon_{p}$ and $N_{\mathrm{smr}}=3$, whereas right panels show data for $2 H_{T}$ with different $N_{\text {smr }}(0$ and 6$)$.

where $\langle\Delta H\rangle$ represents the Monte Carlo average of the change of the Hamiltonian due to the discretized MD integration. Figure 1 compares $N_{\mathrm{MD}, P=0.80}$ and the iteration count for $\mathrm{CG}$ per MD step, denoted by $N_{\text {inv }}$, among the tested formulations. We observe that these two measures of the CPU cost significantly decrease by i) switching from $H_{W}$ to $(2) H_{T}$, ii) switching from $\varepsilon_{Z}$ to $\varepsilon_{p}$, and iii) applying smearing $\left(N_{\mathrm{smr}} \geq 3\right)$. On the other hand, there is no large difference in these measures between Shamir-type kernels $\left(H_{T}\right.$ and $\left.2 H_{T}\right)$ and between $N_{\text {smr }}=3$ and 6.

The product $N_{\mathrm{MD}, P=0.80} N_{\text {inv }}$ can be considered as a measure of the CPU cost per HMC trajectory. As plotted in Fig. 2, the overlap formulation, namely the combination of $H_{W}$ and $\varepsilon_{Z}$, turns out to be computationally very demanding. We can achieve about a factor of 20 acceleration at $M_{\pi} \simeq 400 \mathrm{MeV}$ : a factor of 5 by using $(2) H_{T}$ and $\varepsilon_{p}$, and an additional factor of 4 by smearing. We may expect even bigger gain at smaller quark masses.

These computationally cheaper formulations are, however, off from practical use, if they largely violate chiral symmetry. We compare residual quark mass $m_{\text {res }}$ in Fig. 3. Since the minmax approximation can satisfy $\left|\varepsilon_{Z}(x)\right|^{2} \sim 1$ in its approximation range, $\varepsilon_{Z}$ leads to the least $m_{\text {res }}$ at a given $N_{\mathrm{smr}}$. With our choice of $N_{5}=12$, however, $\left|\varepsilon_{p}(x)\right|^{2}$ largely deviates from unity at $x \lesssim 0.3$, 
Table 2: Status of our simulations at $a^{-1} \simeq 2.4 \mathrm{GeV}$. The third column shows the choice of the MD integrator, namely the leap-frog (LF) or Omelyan (O) integrator. We also list time per HMC trajectory on the whole machine of BlueGene/Q at KEK in the last column.

\begin{tabular}{ll|lllllll}
\hline$a m_{u d}$ & $a m_{s}$ & MD & $N_{\text {MD }}$ & traj & $P$ & $\langle\Delta H\rangle$ & $\left\langle e^{-\Delta H}\right\rangle$ & min/traj \\
\hline \hline 0.019 & 0.040 & LF & 10 & 3000 & $0.78(1)$ & $0.19(1)$ & $0.99(1)$ & 2.7 \\
\hline 0.012 & 0.040 & LF & 13 & 2000 & $0.78(1)$ & $0.17(1)$ & $1.00(1)$ & 3.5 \\
0.012 & 0.040 & O & 3 & 1000 & $0.89(1)$ & $0.07(2)$ & $1.01(1)$ & 2.0 \\
\hline 0.007 & 0.040 & LF & 16 & 1000 & $0.74(1)$ & $0.23(2)$ & $1.04(3)$ & 4.4 \\
0.007 & 0.040 & O & 4 & 2000 & $0.90(1)$ & $0.06(1)$ & $1.00(1)$ & 2.6 \\
\hline \hline 0.019 & 0.030 & LF & 10 & 3000 & $0.79(1)$ & $0.17(1)$ & $1.00(1)$ & 2.8 \\
\hline 0.012 & 0.030 & LF & 13 & 2000 & $0.79(1)$ & $0.14(1)$ & $1.02(2)$ & 3.6 \\
0.012 & 0.030 & O & 3 & 1000 & $0.88(1)$ & $0.10(3)$ & $1.00(2)$ & 2.0 \\
\hline 0.007 & 0.030 & LF & 16 & 2000 & $0.72(1)$ & $0.27(2)$ & $1.00(2)$ & 4.5 \\
0.007 & 0.030 & O & 4 & 1000 & $0.89(1)$ & $0.08(2)$ & $0.99(1)$ & 2.6 \\
\hline
\end{tabular}

where thin-link kernels have many low-lying modes as shown in Fig. 3. Scaling of the kernel $\left(H_{T} \rightarrow 2 H_{T}\right)$ and smearing $\left(N_{\mathrm{smr}}=3\right)$ are very effective to suppress these low-lying modes leading to an order of magnitude smaller $m_{\text {res }}$ compared to the standard domain-wall fermions. Larger $N_{\text {smr }}$ is better in reducing $m_{\text {res }}$ but may distort short distance physics. We refer to Ref. [5] for more detailed discussions.

Figure 4 shows examples of the Monte Carlo history of the topological charge $Q$. A lowlying eigenvalue flips its sign along a tunneling between topological sectors. While scaling and smearing suppress the low-lying modes, the comparison in Fig. 4 suggests that these techniques do not prevent the topological tunneling at $a^{-1} \simeq 2 \mathrm{GeV}$.

From this comparative study, we conclude that the combination of $2 H_{T}$ and $\varepsilon_{p}$ with $N_{\mathrm{smr}}=3$ is the best choice among the tested formulations.

\section{Large-scale simulations}

We have launched large-scale simulations of $N_{f}=2+1$ QCD with good chiral symmetry, namely with $m_{\text {res }}$ well below the physical up and down quark mass $m_{u d, \text { phys. }}$ The tree-level Symanzik gauge action is combined with the fermion formulation chosen by the comparative study to be consistent with our $O\left(a^{2}\right)$-improvement program for heavy quark physics [6]. For controlled continuum and chiral extrapolations, we are planning to simulate the pion masses of 500,400, $300 \mathrm{MeV}$ (and even smaller) at four values of the lattice cut-off $a^{-1} \simeq 2.4,3.0,3.6$ and $4.8 \mathrm{GeV}$. Finite volume effects are suppressed to $1-2 \%$ level by keeping $M_{\pi} L \gtrsim 4$. These simulations are being carried out on BlueGene/Q at KEK (6 racks with a peak speed of 1.258 PFLOPS).

Table 2 shows the current status of our simulations on a $32^{3} \times 64 \times 12$ lattice at $\beta=4.17$, where $a^{-1}$ determined from $r_{0}$ is expected to be $\simeq 2.4 \mathrm{GeV}$. The three values of the bare light quark mass $m_{u d}$ correspond to $M_{\pi} \approx 500,400$ and $300 \mathrm{MeV}$, whereas we take two strange quark masses ( $m_{s}$ 's) near its physical value $m_{s, \text { phys. }}$. We employ the Hasenbusch preconditioning [7] with the mass parameter $a m^{\prime}=0.150$ for two degenerate light flavors, and the rational HMC algorithm [8] for the 
Table 3: Status of our simulations at $a^{-1} \simeq 3.6 \mathrm{GeV}$.

\begin{tabular}{ll|llllll}
\hline$a m_{u d}$ & $a m_{s}$ & $a m^{\prime}$ & $N_{\mathrm{MD}}$ & traj & $P_{\mathrm{HMC}}$ & $\langle\Delta H\rangle$ & $\mathrm{min} / \mathrm{traj}$ \\
\hline \hline 0.0120 & 0.0250 & 0.10 & 4 & 430 & $0.84(2)$ & $0.10(2)$ & 3.6 \\
\hline 0.0080 & 0.0250 & 0.08 & 4 & 330 & $0.85(2)$ & $0.06(2)$ & 4.2 \\
\hline 0.0042 & 0.0250 & 0.04 & 4 & 235 & $0.92(3)$ & $0.04(2)$ & 5.9 \\
\hline \hline 0.0120 & 0.0180 & 0.10 & 4 & - & - & - & - \\
\hline 0.0080 & 0.0180 & 0.08 & 4 & 260 & $0.86(1)$ & $0.05(1)$ & 4.3 \\
\hline 0.0042 & 0.0180 & 0.04 & 4 & 280 & $0.86(3)$ & $0.02(2)$ & 6.0 \\
\hline
\end{tabular}

single strange flavor. We had started our simulations with the simple leap-frog MD integrator, which was later switched to the Omelyan integrator [9] leading to a factor of 2 speed-up. We keep reasonably high acceptance rate $P \simeq 0.7-0.9$ and confirm that $\left\langle e^{-\Delta H}\right\rangle=1$ derived from the area preserving property of $\mathrm{HMC}$ is well satisfied.

We are also carrying out simulations at a larger lattice cut-off $a^{-1} \simeq 3.6 \mathrm{GeV}(\beta=4.35)$ on $48^{3} \times 96 \times 8$. The current status is summarized in Table 3 . We increase the unit trajectory length to $\tau=2$ based on our preparatory study on the auto-correlation (see below). Our choice of the fermion action as well as careful tuning of $m^{\prime}$ at each $m_{u d}$ enable us to achieve the high acceptance rate $P \gtrsim 0.85$ with small $N_{\mathrm{MD}}=4$. We expect half a year to accumulate $10,000 \mathrm{MD}$ time on this large volume by using BlueGene/Q at KEK. This will be accelerated by further optimization of our simulation code [10].

We plot $m_{\text {res }}$ from these simulations in Fig. 5, where the renormalization factor to the $\overline{\mathrm{MS}}$ scheme at $2 \mathrm{GeV}$ is roughly estimated by matching our estimate of the bare value of $m_{s, \text { phys }}$ with a world average [11] in that scheme. It turns out that $m_{\text {res }} \simeq 0.5 \mathrm{MeV}$ at $a^{-1} \simeq 2.4 \mathrm{GeV}$ with $N_{5}=12$. At $a^{-1} \simeq 3.6 \mathrm{GeV}$, $m_{\text {res }}$ is even smaller $(\simeq 0.1 \mathrm{MeV})$ with smaller $N_{5}=8$. While these $m_{\text {res }}$ 's are already much

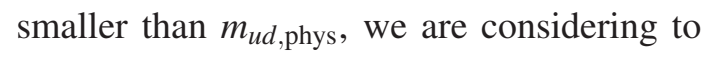
further reduce $m_{\text {res }}$ by reweighting [12].

In Fig. 6, we compare the topological tun-

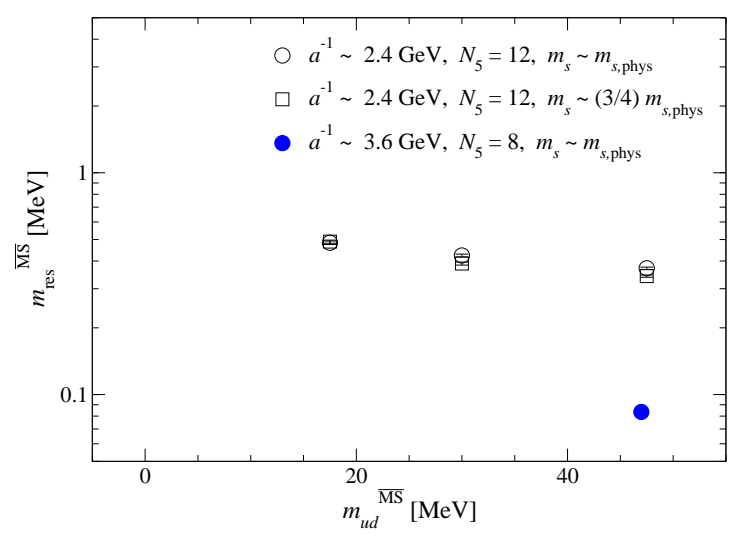

Figure 5: $m_{\text {res }}$ in $\overline{\mathrm{MS}}$ scheme at $2 \mathrm{GeV}$. neling at $a^{-1} \sim 2.4$ and $3.6 \mathrm{GeV}$. The auto-correlation largely increases by approaching the continuum limit with the unit trajectory length $\tau$ held fixed. As suggested in Ref. [13], we observe that topology changes more frequently with larger $\tau$ in our study in quenched QCD at a similar cut-off $a^{-1} \simeq 3.5 \mathrm{GeV}$. This observation leads us to increase $\tau$ when exploring $a^{-1}$ above $2.4 \mathrm{GeV}$ to accelerate our Monte Carlo sampling of topological sectors.

In this article, we reported on our new project of large-scale simulations of $N_{f}=2+1 \mathrm{QCD}$ with good chiral symmetry. The lattice action is chosen by the comparative study to reduce $m_{\text {res }}$ well below the physical quark masses and achieve a factor of 20 acceleration compared to the overlap formulation. We are planning to accumulate high statistics of 10,000 MD time for a precision study 

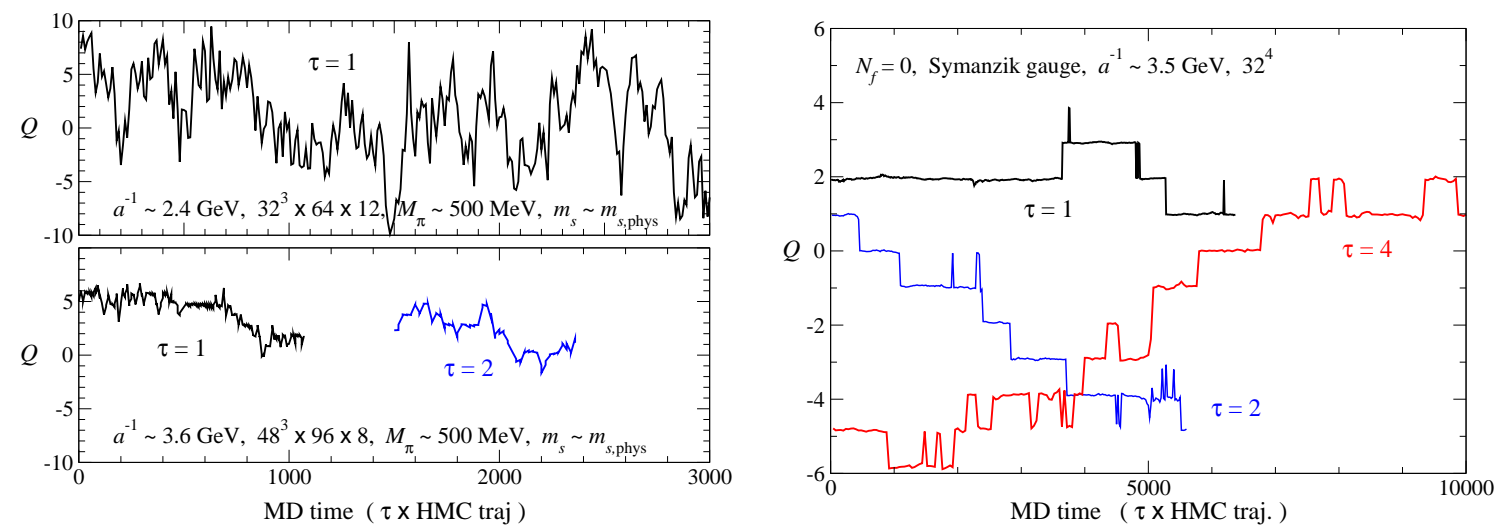

Figure 6: Left panels: Monte Carlo history of topological charge $Q$ in our simulations of $N_{f}=2+1$ QCD at $a^{-1} \simeq 2.4$ (left-top panel) and $3.6 \mathrm{GeV}$ (left-bottom panel). Right panel: history of $Q$ in our study in quenched QCD at $a^{-1} \simeq 3.5 \mathrm{GeV}$ with different values of $\tau$.

of QCD. Our preliminary results on the light hadron physics were presented at this conference [14].

Numerical simulations are performed on Hitachi SR16000 and IBM System Blue Gene Solution at High Energy Accelerator Research Organization (KEK) under a support of its Large Scale Simulation Program (No. 12/13-04). This work is supported in part by the Grants-in-Aid for Scientific Research (No. 21674002, 25287046), the Grant-in-Aid for Scientific Research on Innovative Areas (No. 2004: 20105001, 20105002, 20105003, 20105005, 23105710), and SPIRE (Strategic Program for Innovative Research).

\section{References}

[1] S. Aoki et al. (JLQCD and TWQCD Collaborations), Prog. Theor. Exp. Phys., 01A106 (2012). See also X. Feng, PoS LATTICE 2013, 008; X. Feng et al., ibid., 480; T. Iritani et al., ibid., 376 (in these proceedings).

[2] R.C. Brower, H. Neff and K. Orginos, Nucl. Phys. (Proc.Suppl.) 140, 686 (2005); arXiv:1206.5214 [hep-lat].

[3] C. Morningstar and M. Peardon, Phys. Rev. D 69, 054501 (2004).

[4] C. Aubin et al. (MILC Collaboration), Phys. Rev. D 70, 094505 (2004).

[5] S. Hashimoto et al. (JLQCD Collaboration), PoS LATTICE 2013, 431 (in these proceedings).

[6] Y-G. Cho et al. (JLQCD and UKQCD Collaborations), PoS LATTICE 2013, 255 (in these proceedings).

[7] M. Hasenbusch, Phys. Lett. B 519, 177 (2001).

[8] I. Horváth, A.D. Kennedy and S. Sint, Nucl. Phys. (Proc.Suppl.) 73, 834 (1999).

[9] I.P. Omelyan, I.M. Mryglod and R. Folk, Phys. Rev. E 65, 056706 (2002); T. Takaishi and P. de Forcrand, Phys. Rev. E 73, 036706 (2006).

[10] G. Cossu et al. (JLQCD Collaboration), PoS LATTICE 2013, 482 (in these proceedings).

[11] G. Colangelo et al. (FLAG working group of FLAVIANET), Eur. Phys. J. C 71, 1695 (2011).

[12] H. Fukaya et al. (JLQCD Collaboration), PoS LATTICE 2013, 127 (in these proceedings).

[13] S. Schäfer, R. Sommer, F. Virotta (ALPHA Collaboration), Nucl. Phys. B 845, 93 (2011); Lüscher and Schäfer, JHEP 1107, 036 (2011).

[14] J. Noaki et al. (JLQCD Collaboration), PoS LATTICE 2013, 263 (in these proceedings). 